\title{
ON THE HOMOLOGICAL DIMENSION OF CERTAIN IDEALS
}

\author{
R. E. MACRAE
}

1. Introduction. We present two closely related results connecting homological dimension theory and the ideal theory of noetherian rings. The first, Proposition 4.1, asserts that the only ideals of finite homological dimension in a local ring whose associated prime ideals all have grade one are of the form $a R: b R$. The second, Proposition 4.3 , asserts that if $R$ is a noetherian integral domain, then all such ideals are even projective. The discerning reader will immediately observe that the latter proposition suffices to imply unique factorization in regular local rings. Indeed, our results are to some extent an elaboration of an unpublished proof of unique factorization in regular local rings by Kaplansky. The reader will note that the devices involved in the proof are different from those used by Auslander, Buchsbaum and Nagata in their classic proof of unique factorization as given, for example, in [4].

Of the two propositions outlined above, the assumption in the second that the ring is a domain is to be considered a defect. If we could drop this hypothesis, the first proposition would be superfluous. Indeed, we know of devices whereby it may be dropped for rings of codimension at most three. For larger codimensions, however, the question is quite open.

By way of extension of the above pair of propositions, M. Auslander has shown and will publish elsewhere a proof of the fact that if $R$ is a noetherian integrally closed domain and $A$ is a finitely generated, reflexive $(A=\operatorname{Hom}(\operatorname{Hom}(A, R), R)) R$-module of finite homological dimension and whose endomorphism ring is projective, then $A$ is itself projective.

2. On finitely, freely related projective ideals. In this section we present a criterion, due to Kaplansky, under which a projective ideal is principal.

Definition 2.1. An $R$-module $A$ is said to be finitely, freely related if there exists a finite set $F_{0}, \cdots, F_{n}$ of finitely generated free $R$ modules and homomorphisms such that the sequence $0 \rightarrow F_{n} \rightarrow \cdots$ $\rightarrow F_{0} \rightarrow A \rightarrow 0$ is exact.

Lemma 2.2. If $R$ is any commutative ring and $A$ is a projective, finitely, freely related $R$-module, then there exists a finitely generated

Presented to the Society, November 25, 1960 under the title Homological dimension of ideals in Noetherian domains; received by the editors June 27, 1962. 
free $R$-module, $F$, such that $A \oplus F$ is free.

Proof. Let $0 \rightarrow F_{n} \rightarrow \cdots \rightarrow F_{0} \rightarrow A \rightarrow 0$ be an exact sequence of the type whose existence is guaranteed by the above definition. If $n=0$, then $A$ is free and finitely generated so we are done. For $n>0$ consider $0 \rightarrow F_{n} \rightarrow \cdots \rightarrow F_{1} \rightarrow K \rightarrow 0$ where $0 \rightarrow K \rightarrow F_{0} \rightarrow A \rightarrow 0$. Now $F_{0}$ $\cong K \oplus A$ since $A$ is projective. Further, an induction on the length of the exact sequence gives us a finitely generated free $R$-module, $G$, such that $K \oplus G$ is free and finitely generated. Hence $F_{0} \oplus G$ $\cong(G \oplus K) \oplus A$ and we are done.

Proposition 2.3 (KAPLANSKy). If $I$ is a projective, finitely, freely related ideal of $R$ not consisting entirely of zero divisors, then $I$ is principal and generated by a nondivisor of zero.

Proof. By Lemma 2.2 above, $I \oplus R \oplus \cdots \oplus R \cong R \oplus \cdots \oplus R$. By Lemma 1 of [3], there is an element $a$ of the total quotient ring of $R$ such that $I=a R$. However, $a$ is thus in $I$, so $I$ is principal. We should observe that although Lemma 1 of [3] is stated for domains it is valid, by the same proof, for nondomains when the modules involved contain nondivisors of zero.

3. A stability criterion for grade. We show here that under suitably restrictive hypotheses the grade of a prime ideal remains invariant under localization. For a definition of grade see the appendix.

Proposition 3.1. Let $R$ be a noetherian ring, $A$ a finitely generated $R$-module, $P$ a prime ideal belonging to the null submodule of $A$ and $S$ a multiplicative subset of $R$ disjoint from both $P$ and the annihilator of A. If $\operatorname{dh}(A)<\infty$, then $\operatorname{gr}(P)=\operatorname{gr}\left(P_{S}\right)$.

Proof. Since the annihilator of $A$ is contained in $P$ we have $\operatorname{gr}(P) \leqq \operatorname{gr}\left(P_{S}\right) \leqq \operatorname{gr}\left(P_{P}\right)$ (see Lemma A3). Thus it suffices to show that $\operatorname{gr}(P)=\operatorname{gr}\left(P_{P}\right)$. Let, now, $I$ be an ideal contained in $P$ and generated by a maximal $R$-sequence in $P$. We may then find a prime ideal $Q$ such that $Q$ contains $P$ and belongs to $I$. One easily sees (Lemma A3, again) that the grade of $Q$ is stable under localization. Hence $\operatorname{gr}(P)=\operatorname{gr}\left(P_{Q}\right)=\operatorname{gr}\left(Q_{Q}\right)$. Now, by Proposition A4 and the remarks immediately preceding, we have $\operatorname{gr}\left(P_{Q}\right) \leqq \mathrm{dh}_{R_{Q}}\left(A_{Q}\right) \leqq \operatorname{gr}\left(Q_{Q}\right)$. Combining these inequalities with the equality above yields $\operatorname{gr}(P)=\operatorname{gr}\left(P_{Q}\right)$ $=\mathrm{dh}_{R_{Q}}\left(A_{Q}\right)$. We may combine these equalities with the following inequalities, $\operatorname{gr}\left(P_{Q}\right) \leqq \operatorname{gr}\left(P_{P}\right) \leqq \mathrm{dh}_{R_{P}}\left(A_{P}\right) \leqq \mathrm{dh}_{R_{Q}}\left(A_{Q}\right)$ and thus find that $\operatorname{gr}(P)=\operatorname{gr}\left(P_{Q}\right)=\operatorname{gr}\left(P_{P}\right)$ which completes the proof.

4. On grade one, grade unmixed ideals. Before stating our main results we make a few motivating remarks. In a noetherian ring an 
ideal having the property that each of its prime ideals has grade one is said to be grade one, grade unmixed. An example of such an ideal is one generated by a nondivisor of zero. More generally if $a$ is a nondivisor of zero and $b$ is any element of $R$ not in $a R$, then the ideal of residual quotients $a R: b R$ is also grade one, grade unmixed since its prime ideals are among those which belong to $a R$. Our first result asserts that under certain conditions all grade one, grade unmixed ideals are of this form.

Proposition 4.1. Let $R$ be a noetherian ring and I a grade one, grade unmixed ideal of $R$. If $I$ is finitely freely resolvable, then (i) there exists an element $b$, in the radical of $I I^{-1}$ (where $I^{-1}=\operatorname{Hom}_{R}(I, R)$ ) but not in any of the prime ideals belonging to $I$ and (ii) for any such $b$, there exists an element $a$ in $I$ such that $I=a R: b^{n} R$ for some $n$.

Proof. For the first part of the result we note that if $\sqrt{ }\left(I I^{-1}\right)$ is contained in the union of the prime ideals belonging to $I$ then, by a standard argument, it is contained in one of them, say $P$. By Proposition 3.1 we may assume (by localizing) that $R$ is local and $P$ is its maximal ideal. Since $\operatorname{dh}(R / I) \leqq \operatorname{gr}(P)$ (see [1]) and $\operatorname{gr}(P)=1$, it is clear that in this case $I$ is both projective and noninvertible which is certainly impossible. Pick now any element $b$ in $\sqrt{ }\left(I I^{-1}\right)$ but not in any of the prime ideals of $I$. Consider the multiplicative set $S=\left\{1, b, b^{2}, \cdots, b^{n}, \cdots\right\}$. It is clear that the extension, $I_{S}$, of $I$ to the quotient ring $R_{S}$ is $R_{S}$-projective and finitely, freely resolvable. Hence by Proposition 2.3 it is $R_{S}$-principal. Thus there is an element $a$, in $I$ such that $b^{n} I \leqq a R$ for some $n$, i.e., $I \leqq a R: b^{n} R$. Now the fact that $b$ is not in any of the prime ideals of $I$ implies that $a R: b^{n} R \leqq I$. Hence $I=a R: b^{n} R$ and we are done.

For our second main result the following useful lemma is crucial.

LEMMA 4.2 (NAGATA). If $R$ is an integral domain and $b, a$ is an $R$ sequence in some proper ideal of $R$, then $a+b x$ generates a prime ideal in the polynomial ring $R[x]$.

Proof. Suppose $f(x) g(x)=h(x)(a+b x)$ for appropriate $f(x), g(x)$ and $h(x)$ in $R[x]$. We must show that either $f(x)$ or $g(x)$ is a multiple of $a+b x$. By the so-called Generalized Euclidean Algorithm $b^{m} f(x)$ $=q(x)(a+b x)+c$ and $b^{n} g(x)=r(x)(a+b x)+d$ where $m$ and $n$ are integers, $q(x)$ and $r(x)$ are in $R[x]$ and $c$ and $d$ are in $R$, all appropriately chosen. Hence

$$
b^{m+n} h(x)(a+b x)=[q(x) r(x)(a+b x)+q(x) d+r(x) c](a+b x)+c d .
$$

Thus $c d=0$. Since $R$ is an integral domain, either $c$ or $d$ vanishes; let us say $c=0$. Hence $b^{m} f(x)=q(x)(a+b x)$. By an easy application of 
the assumption that $b, a$ forms an $R$-sequence we find that $q(x)$ $=b q_{1}(x)$ for some $q_{1}(x)$ in $R[x]$. Hence $b^{m-1} f(x)=q_{1}(x)(a+b x)$. Continuing in this way we obtain, finally, $f(x)=q_{m}(x)(a+b x)$.

Proposition 4.3. Let $R$ be a noetherian integral domain and $I$ a grade one, grade unmixed ideal of $R$. If I has finite homological dimension, then $I$ is projective.

Proof. If $I I^{-1}=R$ we are done; so let us suppose $I I^{-1}$ is a proper ideal. We claim $\operatorname{gr}\left(I I^{-1}\right) \geqq 2$. If not $I I^{-1}$ is contained in some prime ideal $P$ which belongs to a principal ideal. Localizing at $P$ gives us a local ring whose maximal ideal has grade one. As in the first part of the proof of the preceding proposition, this is impossible. By localizing at some proper prime ideal containing $I I^{-1}$ we may assume $R$ to be a local ring and thus $I$ finitely freely resolvable. Consider the polynomial ring $R[x]$ and the extended ideal $I^{*}$ consisting of all polynomials in $R[x]$ having all of their coefficients in $I$. We observe that $I^{*}$ satisfies all of the hypotheses of the proposition relative to $R[x]$ and, further, that $I^{*}$ is not invertible in $R[x]$. By Lemma 4.2, $I^{*} I^{*-1}$ contains an element $p(x)$ which generates a prime ideal. Moreover, the construction of $p(x)$ is easily seen to preclude the possibility that $p(x)$ is contained in any of the prime ideals belonging to $I^{*}$ (these are, after all, only the extensions of the prime ideals of $I$ to $R[x])$. Hence by the second part of Proposition 4.1, $I^{*}=f(x) R[x]: p^{n}(x) R[x]$ for some $n$ and some $f(x)$ in $R[x]$. But the primeness of $p(x)$ implies that $f(x), p^{n}(x)$ is an $R$-sequence, i.e., $I^{*}$ is generated by $f(x)$ which thus makes $I^{*}$ invertible, which is a contradiction.

COROLlaRY 4.4. If $R$ is a noetherian domain and $I$ is an ideal of finite homological dimension generated by two elements, then $\operatorname{dh}(I) \leqq 1$.

Proof. Let $I=a R+b R$. The result follows from the proposition above and the exact sequence $0 \rightarrow a R: b R \rightarrow R \oplus R \rightarrow a R+b R \rightarrow 0$.

COROLlaRY 4.5. A regular local ring is a unique factorization domain.

Proof. By Theorem 1.10 of [1] a regular local ring has finite global dimension. Hence, by the Proposition 4.3, above, all the grade one prime ideals of the ring are principal. This is easily seen to imply unique factorization.

Appendix. In this section we present a quite short proof of a wellknown result of Rees. Unless otherwise stated, $R$ will denote a noetherian ring and $A$ a finitely generated $R$-module which is nonnull.

Definition A1. A finite, ordered set $a_{1}, \cdots, a_{n}$ of elements of a 
proper ideal, $I$, of $R$ is said to be an $A$-sequence in $I$ if $a_{i}$ is not a zero divisor on the factor module $A / a_{1} A+\cdots+a_{i-1} A$ for $i=1, \cdots, n$. (The case when $i=1$ is interpreted to mean that $a_{1}$ is not a zero divisor on $A$ itself.) The integer $n$ is called the length of the $A$-sequence and the $A$-sequence is said to be maximal if $I$ consists entirely of zero divisors on $A / a_{1} A+\cdots+a_{n} A$.

It may be shown (see, for example [2]) that for a fixed $I$ and $A$ any $A$-sequence in $I$ can be extended to a maximal one and any two maximal ones have the same length. This fact gives rise to the following

Definition A2. The common length of maximal $A$-sequences in $I$ is called the grade of $I$ on $A$ and is written $\operatorname{gr}(I, A)$. In keeping with current terminology we write $\operatorname{gr}(I)$ when $A=R$ and $\operatorname{codh}(A)$ when $R$ is local and $I$ is the maximal ideal.

The reader may easily supply for himself the proof of the following lemma.

Lemma A3. Suppose $S$ is a multiplicative set in $R$ disjoint from both $I$ and the annihilator of $A$. Then $\operatorname{gr}(I, A) \leqq \operatorname{gr}\left(I_{S}, A_{S}\right)$ where $I_{S}$ and $A_{S}$ are the extensions relative to the quotient ring $R_{S}$.

In [1] Auslander and Buchsbaum have shown if $R$ is local and $A$ has finite homological dimension, then $\operatorname{dh}(A) \leqq \operatorname{gr}(M)$ where $M$ is the maximal ideal of $R$ and $\operatorname{dh}(A)=\operatorname{gr}(M)$ if and only if $M$ belongs to the null submodule of $A$. Using this we may give a quite short proof of the following well-known result of Rees.

Proposition A4. If $P$ is a prime ideal which belongs to the null submodule of $A$, then $\operatorname{gr}(P) \leqq \operatorname{dh}(A)$.

Proof. When $\operatorname{dh}(A)=\infty$, the assertion is vacuous so let us assume that $A$ has finite homological dimension. By Lemma A3, $\operatorname{gr}(P)$ $\leqq \operatorname{gr}\left(P_{P}\right)$. Further, it is well known that $\operatorname{dh}_{R_{P}}\left(A_{P}\right) \leqq \mathrm{dh}_{R}(A)$. Now by the observation immediately preceding this proposition we have $\operatorname{gr}\left(P_{P}\right)=\mathrm{dh}_{R_{P}}\left(A_{P}\right)$. Hence, $\operatorname{gr}(P) \leqq \mathrm{dh}(A)$.

\section{REFERENCES}

1. M. Auslander and D. Buchsbaum, Homological dimension in local rings, Trans. Amer. Math. Soc. 85 (1957), 390-405.

2. - Codimension and multiplicity, Ann. of Math. (2) 68 (1958), 625-657.

3. I. Kaplansky, Modules over dedekind rings and valuation rings, Trans. Amer. Math. Soc. 72 (1952), 327-340.

4. O. Zariski and P. Samuel, Commutative algebra, Vols. 1, 2, Van Nostrand, Princeton, N. J., 1958, 1960.

UNiversity of Chicago 